\title{
ON THE RELIABILITY OF ACCELERATED TESTING IN AIGaAs/InGaAs/GaAs PHEMTs
}

\author{
K. F. YARN ${ }^{\mathrm{a} *}$, W. C. CHIEN ${ }^{\mathrm{a}}$ and C. S. WANG ${ }^{\mathrm{b}}$ \\ ${ }^{a}$ Far East College, Department of Electrical Engineering, Optoelectronic Semiconductor Center, \\ Hsin-Shih, Tainan 744, Taiwan, Republic of China; ${ }^{\mathrm{b}}$ National Cheng Kung University, \\ Department of Electrical Engineering, Tainan, Taiwan 701, Republic of China
}

(Received 7 November 2002; In final form 17 November 2002)

\begin{abstract}
The study of different stress on device characteristics of AlGaAs/InGaAs/GaAs PHEMTs has been researched and developed in this report. Many catastrophic degradation mechanisms such as hot-electron, gate-drain breakdown, $I_{D S}, I_{G}, V_{P}$ and gate Schottky barrier effects are discussed in detail. In addition, the accelerated testing of the temperature-dependent effects on $I_{D}, G_{m}$ and Schottky barrier are examined.
\end{abstract}

Keywords: PHEMT; Stress

\section{INTRODUCTION}

While hot-electron related reliability issues have been widely studied in Si MOS devices and integrated circuits, the behavior of compound semiconductor FETs is much less assessed. In particular, devices such as $\mathrm{AlGaAs} / \mathrm{InGaAs} / \mathrm{GaAs}$ pseudomorphic high electron mobility transistors (PHEMTs) have been proved successful in a variety of applications, including low noise amplifier at Q-band [1], V-band monolithic power amplifiers [2], and MMIC power amplifiers at Ka-band [3] and W-band [4]. The use of the AlGaAs/InGaAs heterojunction in PHEMTs provides several advantages over MESFETs and conventional AlGaAs/GaAs HEMTs, and the advantages include: (1) larger conduction band discontinuity, which ensures better electron confinement; (2) the excellent electronic transport properties of InGaAs, which allow improved frequency performances. However, the InGaAs channel layer has a small band-gap, which favors impact ionization. Thus, the effects of hot-electron and impact ionization on device reliability need to be studied.

Since the behavior of the Schottky barrier under hot-electron test is also much less discussed. In this work, the mechanisms for the Schottky barrier change before and after hot-electron stress are examined. We have also performed measurements of other DC characteristics to investigate further the change of the device characteristics under hotelectron test. In addition, we will probe into temperature effects of device characteristics because it plays an important part during its operation. We also discuss the hot-electron

* Current address: P.O. Box 345, Tainan 704, Taiwan, Republic of China. E-mail: ymo86@yahoo.com.tw 
related phenomena in AlGaAs/InGaAs/GaAs PHEMTs, i.e. breakdown walkout in PHEMTs [5]. This phenomenon is a creation and filling of electron traps by hot carriers in the gate-drain region [6,7]. The temperature-dependent characteristics of AlGaAs PHEMTs with temperature ranging from $300 \mathrm{~K}$ to $420 \mathrm{~K}$ with step of $30 \mathrm{~K}$ will be studied.

\section{RESULTS AND DISCUSSION}

\subsection{The AlGaAs/InGaAs/GaAs PHEMT Structure}

The AlGaAs/InGaAs/GaAs structure consists of a 3000 A-thick undoped GaAs buffer layer, a 150 A-thick InGaAs undoped channel layer, a 20 A-thick undoped AlGaAs spacer layer, a 200 A-thick $n$-type AlGaAs donor layer, and a 300 A-thick heavily doped cap layer. The Hall mobility at room temperature and $77 \mathrm{~K}$ are $5650 \mathrm{~cm}^{2} / \mathrm{V}$-sec and $17,300 \mathrm{~cm}^{2} / \mathrm{V}$-sec, corresponding to the two-dimension carrier densities $2.46 \times 10^{12} \mathrm{~cm}^{-2}$ and $2.03 \times 10^{12} \mathrm{~cm}^{-2}$, respectively. The gate dimension of the devices is $0.25 \mu \mathrm{m} \times 160 \mu \mathrm{m}$. The schematic crosssectional view of the $\mathrm{AlGaAs} / \mathrm{InGaAs} / \mathrm{GaAs}$ structure is shown in Figure 1(a).

\subsection{Hot-electron Tests of AlGaAs PHEMT}

\subsubsection{Degradation due to Hot-electron and Impact Ionization}

Hot-electron (HE) and impact ionization (II) play important roles in the reliability of PHEMTs devices, since: (1) the gate must be very short $(L g \leq 0.25 \mu \mathrm{m})$ for higher frequency applications, therefore the fields soar to very high values for relatively low drain bias; (2) the device must be biased deep into saturation to get higher output power; (3) the channel layer must have a small band-gap for good confinement, which favors impact ionization; (4) the large mobility makes the channel electrons easily quit to heat up. Hence, it is necessary to study PHEMT reliability from HE and II standpoints. Figure 1(b) shows the HE-related phenomena in an AlGaAs PHEMT.

In the InGaAs channel layer, impact ionization is triggered when a hot-electron scattering with a lattice atom may provide a valence band electron with sufficient energy to leap into the conduction band. Some of the holes generated by impact ionization overcome the valence band discontinuity entering the donor layer, may be collected by the gate electrode and result in an increase of gate current, may be trapped in $\mathrm{AlGaAs} / \mathrm{InGaAs}$ heterojunction or under the gate electrode, causing a degradation in transconductance $\left(G_{m}\right)$, drain current $\left(I_{D}\right)$, and pinchoff voltage $\left(V_{p}\right)$.

\subsubsection{Gate-drain Breakdown Walkout}

Gate-drain breakdown (GDB) is the main factor limiting the power-handling capability of compound semiconductor heterostructure FETs, which are biased in the saturation region in most analog applications. The $I-V$ characteristics of AlGaAs PHEMTs before and after stress for 60 minutes at $V_{D S}=7 \mathrm{~V}, V_{G S}=0 \mathrm{~V}$ are shown in Figure 2. It should be noted that the stress has induced a significant drop of the drain current $I_{D S}$ at high drain voltage, and it is so called breakdown walkout, which is attributed to hot-electron related effects reported by Menozzi. The gate-drain breakdown walkout is believed to be caused by creation and filling of electron traps in the gate-drain region and by attendant widening of the depletion region, that results in a reduced electrical field and increased breakdown voltage $[8,9]$. End resistance measurements [10] indicate that the drain-to-channel resistance $R_{D}$ increases after a hot-electron stress, $R_{D}$ is increased by about $12 \%$ after the stress, while on the source 


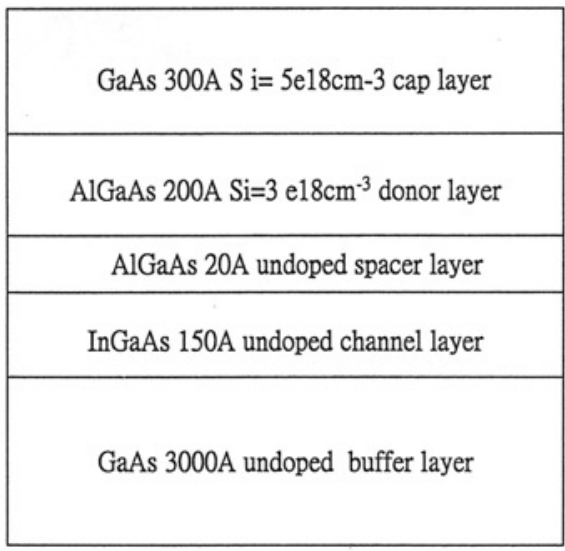

(a)

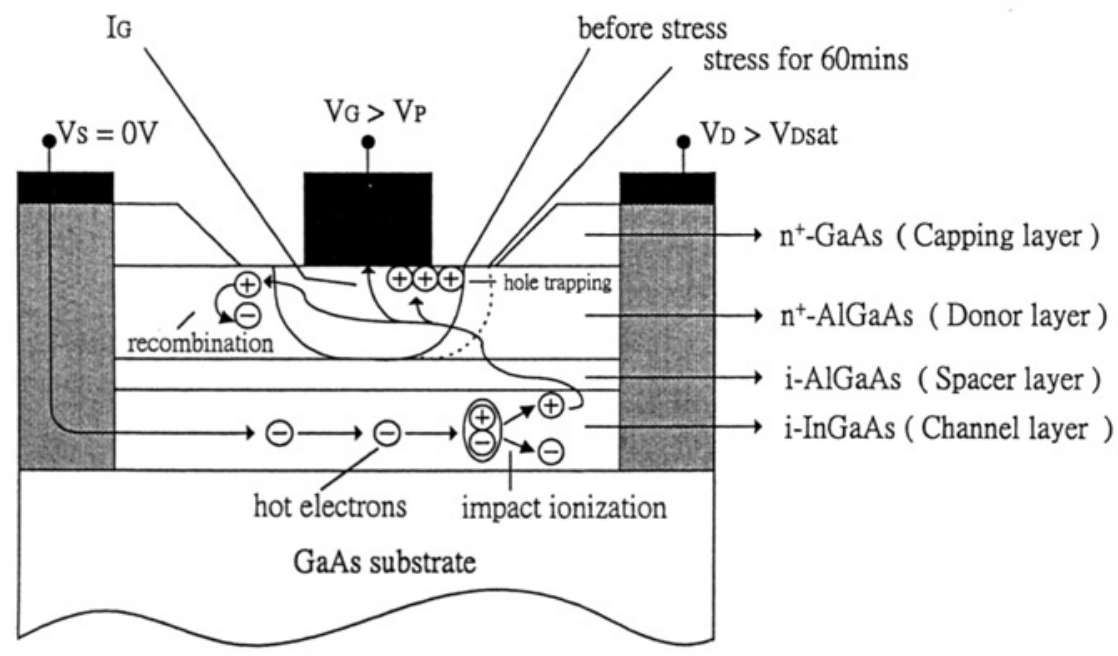

(b)

FIGURE 1 (a) The schematic cross-sectional view of the AlGaAs/InGaAs/GaAs structure. (b) The HE-related phenomena in a AlGaAs/InGaAs/GaAs PHEMT.

side only a slight decrease $(0.2 \%)$ of $R_{S}$ is measured. The $R_{D}$ increase is consistent with the hypothesis of the widening of the depletion in the gate-drain region, and a slight decrease of $R_{S}$ indicates the increase of $I_{D S}$ in the saturation region may be due to the effect of pinch-off voltage shift, to be discussed later on. In addition, it deserves to be mentioned that the gatedrain breakdown walkout is permanent. It does not recover by storing the device for a long time after the stress.

\subsubsection{Existence of the Kink}

A kink is observed at $V_{D S} \sim 2.5 \mathrm{~V}$ before and after the stress as reported in Figure 2, indicating that trapping-detrapping phenomena are present [11-13]. It should be noted that the kink 


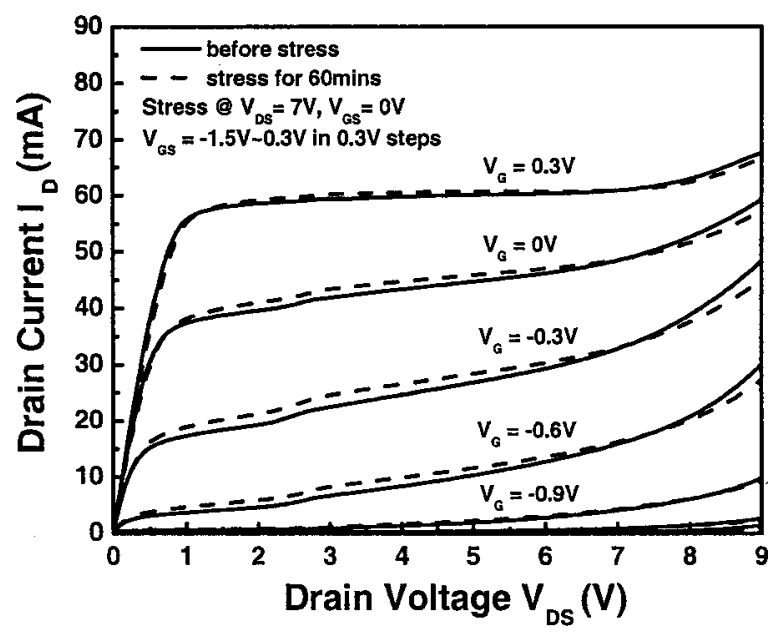

FIGURE 2 The $I-V$ characteristics of AlGaAs/InGaAs/GaAs PHEMTs before and after stress for 60 minutes at $V_{D S}=7 \mathrm{~V}, V_{G S}=0 \mathrm{~V}$.

position shifts towards higher $V_{D S}$ after the stress. This is because the stress induced a decrease of the pinch-off voltage, which meant that, at the same $V_{G S}$ value, the maximum field in the channel is lower in the stressed device than it is in the unstressed one [14].

\subsubsection{An Increase of $I_{D S}$ in the Saturation Region}

Figure 2 indicates also that the hot-electron stress has yielded an increase of $I_{D S}$ in the saturation region, the physical mechanism is a reduction of negative charge in deep traps and DXcenters or an accumulation of positive charge located under the gate, which lead to pinch-off voltage shifts towards further negative $V_{G S}$ values, as shown in Figure 3. If $\Delta I_{D S}$ is due to a shift in the pinch-off voltage $\Delta V p$, then the correlation between $\Delta I_{D S}$ and $\Delta V p$ can be

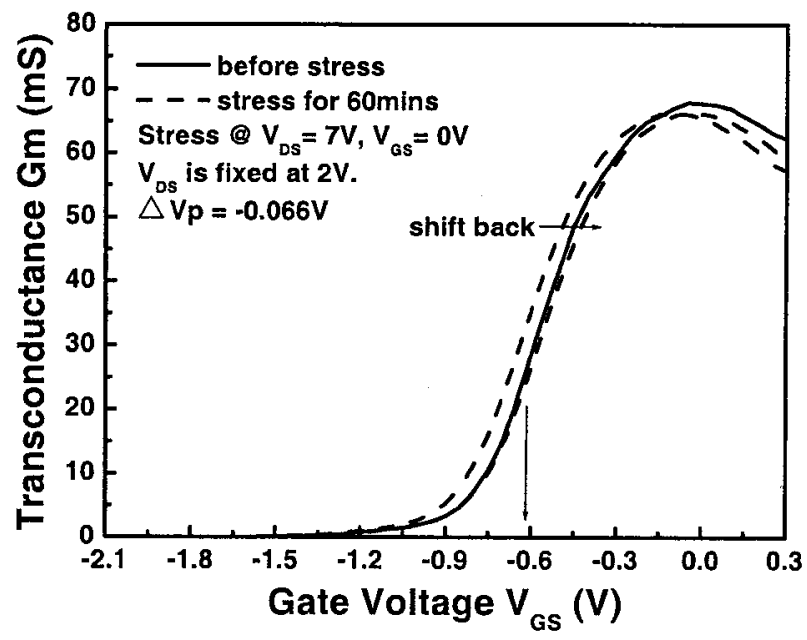

FIGURE 3 The transfer curve of $\mathrm{AlGaAs} / \mathrm{InGaAs} / \mathrm{GaAs}$ PHEMTs before and after stress for 60 minutes at $V_{D S}=7 \mathrm{~V}, V_{G S}=0 \mathrm{~V}$. 
explained according to the following simple model: $\Delta I_{D S}\left(\Delta V_{G S}\right)=I_{D S}\left(V_{G S}-\Delta V p\right)-$ $I_{D S}\left(V_{G S}\right)$. Substituting $I_{D S}\left(V_{G S}-\Delta V p\right)$ with its first Taylor polynomial term, we can obtain $I_{D S}\left(V_{G S}-\Delta V p\right)=I_{D S}\left(V_{G S}\right)-G_{m}\left(V_{G S}\right) \Delta V p$; hence $\Delta I_{D S}\left(V_{G S}\right)=-G_{m}\left(V_{G S}\right) \Delta V p$ [15]. If we use the model to fit our experimental data (see Fig. 4), the result is different from [15]. The possible explanations are: (1) short channel effects arise and mix up with the pinch-off voltage degradation; (2) there are more interface states present at AlGaAs/InGaAs heterojunction due to generation by hot carrier bombardment, which causes the channel carrier density reduction due to the charges trapping and mobility decrease due to the addition surface scattering, inducing the $I_{D S}$ and $G_{m}$ degradation. Hence, the effects of carriers trapped between $\mathrm{AlGaAs} / \mathrm{InGaAs}$ heterojunction mix up with the pinch-off voltage shift effect. The last phenomenon is probably the dominant one, since the maximum $G_{m}$ is slightly decreased after the stress (see Fig. 3). In addition, if we shift the stressed transfer curve back (see Fig. 3) and compare with Figure 4, we can find that at $V_{G S}<-0.6 \mathrm{~V}$, after the hot-electron stress the shape of $G_{m}$ is almost unchanged (see Fig. 3) and the fit line is also perfect (see Fig. 4). However, at $V_{G S}>-0.6 \mathrm{~V}$, the phenomena behave in the opposite way. This is because the effects of the addition surface scattering are more and more evident with further increasing $V_{G S}$ towards positive values and mixing up with the pinch-off voltage shift effect. Hence, when $V_{G S}>-0.6 \mathrm{~V}$, the shape of $G_{m}$ and the fit line begin to degrade (see Figs. 3 and 4 ) after the stress.

\subsubsection{A Bell-shaped Behavior of $I_{G}$}

Figure 5 shows $I_{G}$ as a function of $V_{G S}$ at various $V_{D S}$ from $5.5 \mathrm{~V}$ to $7.5 \mathrm{~V}$, in $0.5 \mathrm{~V}$ steps. $I_{G}$ presents a typical bell-shaped behavior previously observed in MESFETs [16] and AlGaAs/GaAs HEMTs [17]. At $V_{G S}<-1.2 \mathrm{~V}, I_{G}$ is dominated by reverse current and $I_{D}$ becomes negligible since the device is in pinch-off condition. In fact, the reverse leakage current mechanisms include mainly (1) thermionic emission; (2) thermionic field emission; (3) field emission. Thermionic emission reverse leakage current is affected by image-force barrier height lowering, and both thermionic field emission and field emission are tunnelling mechanisms that depend on Schottky barrier width and height [18].

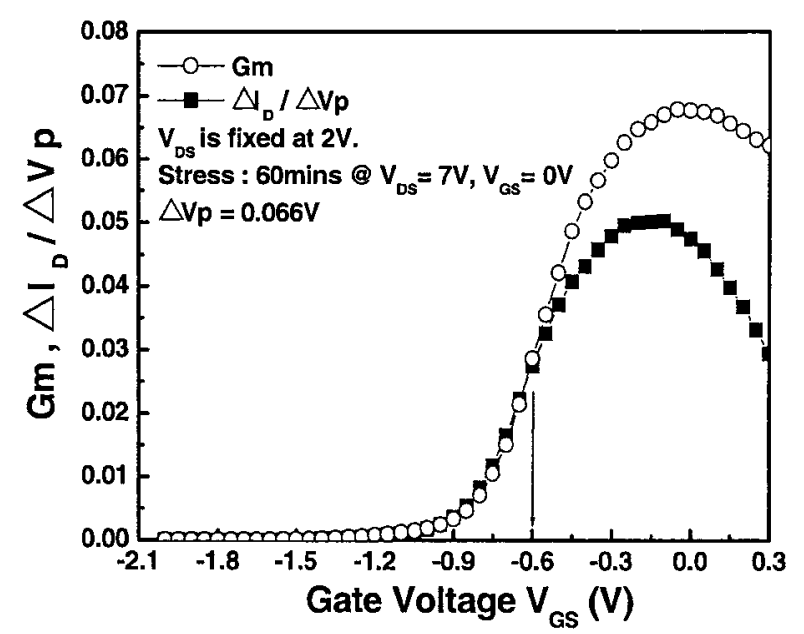

FIGURE $4 \Delta I_{D S}$ and $-G_{m} \Delta V_{p}$ as a function of $V_{G S}$. The $\Delta I_{D S}$ and $G_{m}$ have been calculated at $V_{D S}=2 \mathrm{~V} . \Delta I_{D S}$ is the $I_{D S}$ current change after 60 minutes of stress at $V_{D S}=7 \mathrm{~V}, V_{G S}=0 \mathrm{~V}$. 


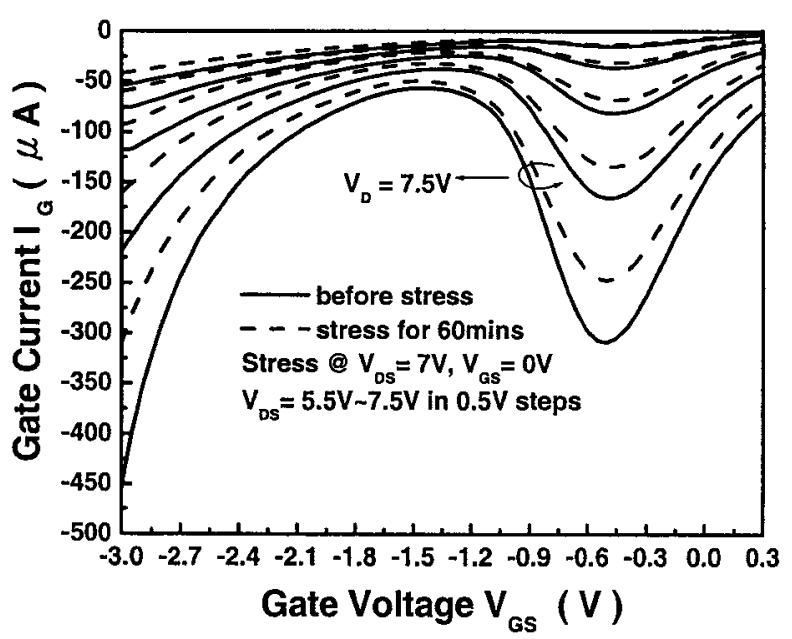

FIGURE 5 Gate current versus gate bias measured at different $V_{D S}$ values of AlGaAs/InGaAs/GaAs PHEMTs, before (solid line) and after (dashed line) stressing at $V_{D S}=7 \mathrm{~V}, V_{G S}=0 \mathrm{~V}$ for 60 mins.

The relationship between reverse leakage current density and Schottky barrier height is [19]

$$
J_{r}=A * T^{2} \exp \left(\frac{-q \phi_{B}}{k T}\right)\left[\exp \left(\frac{q V}{k T}\right)-1\right]
$$

where $J_{r}$ is the reverse leakage current density, and $\phi_{B}$ is the Schottky barrier height.

The thermionic emission reverse leakage current is affected by image-force barrier height lowering, and the image-force barrier height lowering depends on the electric field at metal-semiconductor interface and it can be written as

$$
\Delta \phi_{B}=\left[\frac{q E_{m}}{4 \pi \varepsilon s}\right]^{1 / 2}
$$

where $\Delta \phi_{B}$ is the image-force barrier height lowering, and $E_{m}$ is the electric field at the metal-semiconductor interface.

Then the thermionic emission reverse leakage current density, accounting for image-force barrier height lowering may be expressed as

$$
J_{r} \sim A * T^{2} \exp \left(\frac{-q \phi_{B}}{k T}\right) \exp \left(\frac{q \Delta \phi_{B}}{k T}\right)
$$

if we assume the metal-semiconductor Schottky barrier may be approximated as a triangular barrier as shown in Figure 6(a). Then, the slope of the triangular potential barrier is the electric field at the metal-semiconductor interface, and the electric field at the metal-semiconductor interface will rise with increasing $V_{G S}$ towards negative values. Hence, the image-force height lowering increases with increasing $\left|V_{G S}\right|$ values (see Eq. (2)). From Eq. (3), we can know that the thermionic emission reverse leakage current will increase with increasing $\left|V_{G S}\right|$ values due to an increase of image-force barrier height lowering decreasing the effective Schottky barrier height. Furthermore, the tunnelling current increases with increasing $\left|V_{G S}\right|$ values (since barrier width and height reduce with increasing $\left|V_{G S}\right|$ values). 


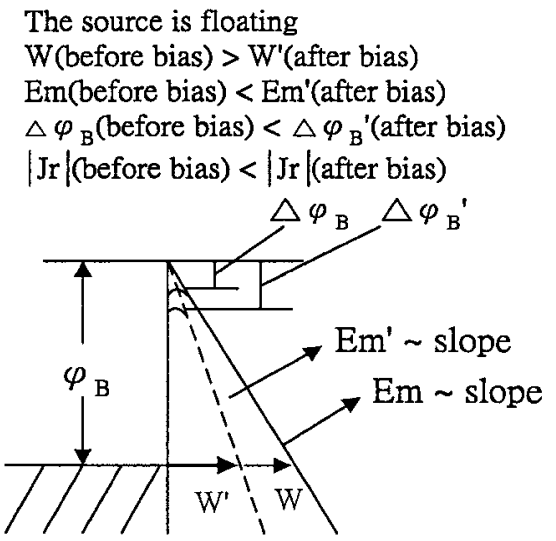

(a)

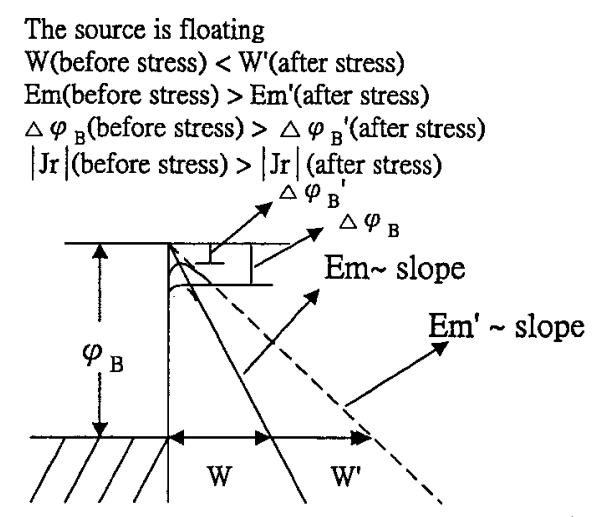

(b)

FIGURE 6 (a) Triangular barrier approximation for a metal-semiconductor barrier, before (solid line) bias and after (dashed line) reverse bias. The source is floating. (b) Triangular barrier approximation for a metalsemiconductor barrier, before (solid line) and after (dashed line) stressing. The source is floating.

Consequently, at $V_{G S}<-1.2 \mathrm{~V}$ region, the reverse leakage current increases with increasing $V_{G S}$ towards negative values.

At $V_{G S}>-1.2 \mathrm{~V}$, the mechanisms are similar to the previous report [20]. $I_{D}$ increases with increasing $V_{G S}$ towards positive values and there are more and more electrons transferring in the high-field region between gate and drain, which makes the impact ionization rate rise. The holes generated by impact ionization will be collected by gate electrode and therefore $\left|I_{G}\right|$ increases up to $V_{G S}=-0.5 \mathrm{~V}$ (see Fig. 5). However, at $V_{G S}>-0.5 \mathrm{~V}$ region, the longitudinal electrical field in the gate-drain region decreases with further increasing $V_{G S}$ towards positive values. In consequence, $\left|I_{G}\right|$ decreases gradually again.

After 60 minutes of hot-electron stress at $V_{D S}=7 \mathrm{~V}, V_{G S}=0 \mathrm{~V},\left|I_{G}\right|$ reduces for all different $V_{G S}$ values at different $V_{D S}$ steps $\left(V_{D S}=5.5 \mathrm{~V}\right.$ to $7.5 \mathrm{~V}$ in $0.5 \mathrm{~V}$ steps), as reported in Figure 5. These possible explanations are: (1) the depletion under the gate expands towards the drain after the stress, and the widening of the depletion will result in reducing the maximum electrical field $[21,22]$ and make the impact ionization rate decrease. Thus, $\left|I_{G}\right|$ decreases at $-1.2 \mathrm{~V}<V_{G}<-0.5 \mathrm{~V}$ region after the stress; (2) at $V_{G}<-1.2 \mathrm{~V}$ region, 
the decrease of reverse leakage current is also due to, after the stress, the widening of the depletion in the gate-drain region, which results in the image-force barrier height lowering decrease due to the electric field at the metal-semiconductor interface reducing and the tunneling current reduction due to the barrier width increasing (see Fig. 6(b)). In consequence, $\left|I_{G}\right|$ decreases at $V_{G}<-1.2 \mathrm{~V}$ region after the stress. Equally, the reverse current decreases at $V_{G}>-0.5 \mathrm{~V}$ region after the stress; (3) the resistance of the drain access region $\left(R_{D}\right)$ increases by about $12 \%$ after the stress, it is consistent with the hypothesis of the widening of the depletion in gate-drain region. In addition, we have also measured reverse bias characteristics of the gate-drain diode with source floating, as shown in Figure 7(a), in order to further identify that the depletion in the gate-drain region is widened by the hot-electron stress. Figure 7(a) shows that the reverse current of the gate-drain diode with source floating decreases after a hot-electron stress of $60 \mathrm{mins}$ at $V_{D S}=7 \mathrm{~V}$ and $V_{G S}=0 \mathrm{~V}$, indicating the image-force barrier height lowering decreases after the stress (the decrease of the imageforce barrier height lowering is due to the widening of the depletion in the gate-drain region, see Figure 6(b) and Eq. (3)). Thus, the depletion in the gate-drain region is widened certainly by the stress.

\subsubsection{A Further Discussion of the Schottky Junction}

After a hot-electron stress, there are two main mechanisms that will influence the characteristics of Schottky barrier height. One is the effect of the depletion widening, and the other is the effect of the carriers trapping under the gate. The widening of the depletion under the gate can cause a reduced peak electric field $[22,23]$ and a decrease of image-force barrier height lowering (see Eq. (2)), which results in a larger effective Schottky barrier height and a smaller reverse leakage current density (see Eq. (3)). On the contrary, the carriers trapping under the gate may cause an increase of the electric field at metal-semiconductor interface and result in a smaller effective Schottky barrier height due to the image-force barrier height lowering increasing (see Eq. (2)). Hence, if we measure the reverse bias characteristics of the

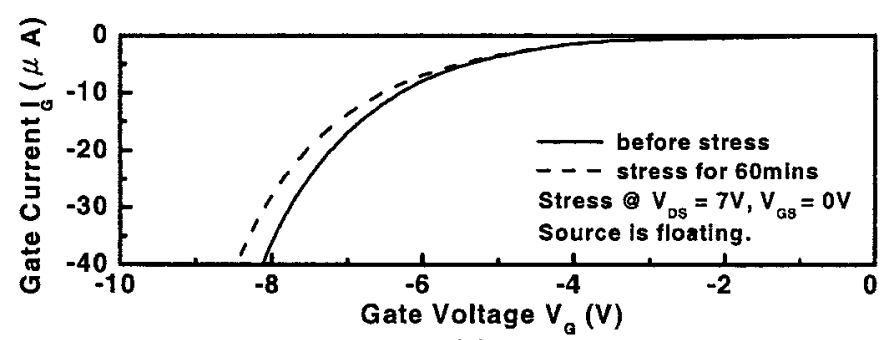

(a)

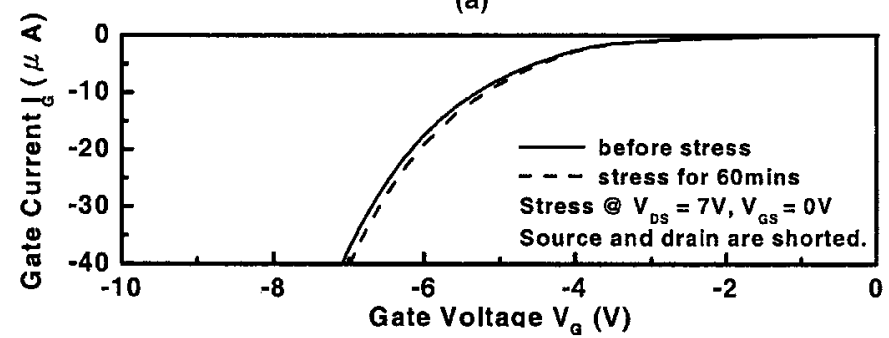

(b)

FIGURE 7 The reverse bias characteristics of the gate-drain diode of InGaP PHEMTs. (a) The source is floating. (b) The source and drain are shorted. 
Schottky junction with source and drain shorted, we can find that after the hot-electron stress, the case is opposite to the reverse bias characteristics of the Schottky junction with source floating, as reported in Figure 7(b). These possible explanations are: (1) when the source is floating, the effect of the depletion widening dominates the device characteristics, and results in a larger effective Schottky barrier height and smaller reverse leakage current after the stress (see Fig. 7(a)); (2) when source and drain are shorted, we not only consider the effect of the carriers trapping in the gate-drain region, but also in the gate-source region. Therefore, the effect of the carrier trapping becomes the dominant mechanism, which will result in a smaller effective Schottky barrier height and a larger reverse leakage current after a hot-electron stress (see Fig. 7(b)).

\subsection{Temperature-dependent Characteristics of AlGaAs PHEMTS}

PHEMT power amplifiers play an important role in microwave-integrated circuits. A huge amount of heat will be generated because they consume a lot of dc power; this influences the performance and the reliability of devices and circuits. Therefore, the study of the change in device DC characteristics under different operational temperatures allows us to understand further the thermal behavior of the devices. In addition, knowledge of the temperature in DC characteristics of PHEMTs is essential to be able to improve device reliability and to optimize device design and performance. In this section, we get an understanding of the influence of temperature on DC characteristics of AlGaAs PHEMTs and allow one to investigate the possibility of improving device stability.

\subsubsection{Thermal Effects of $I_{D}$ and $G_{m}$ in the Saturation Region}

The $I-V$ characteristics and transfer curve of AlGaAs PHEMTs with different temperature ranging from $300 \mathrm{~K}$ to $420 \mathrm{~K}$ with steps of $30 \mathrm{~K}$ are shown in Figures 8 and 9 . At lower $V_{G S}$ values, an increase of the $I_{D}$ and $G_{m}$ in the saturation region is due to a slight increase of the magnitude of the pinch-off voltage with increasing temperature. The correlation between pinch-off voltage and temperature is shown in Figure 10, and the magnitude of the pinch-off voltage increase with increasing temperature may be due to the trapped electrons compensated by holes generated by heat or an accumulation of holes located under the gate. At higher $V_{G S}$ values, however, the $I_{D}$ and $G_{m}$ begin to degrade with increasing

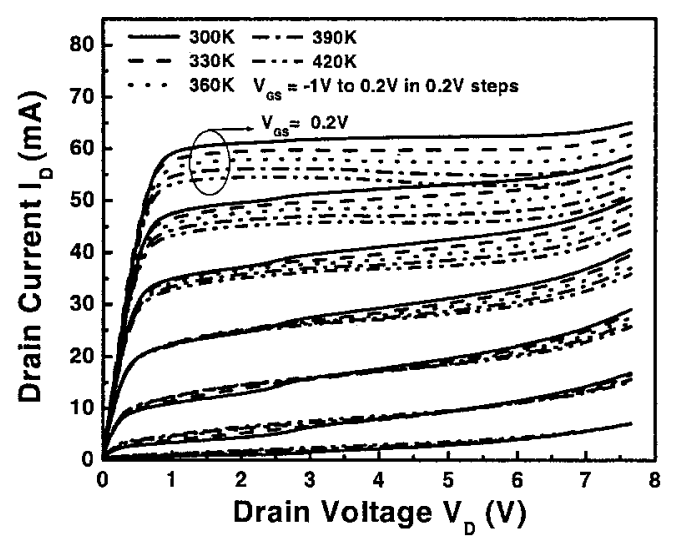

FIGURE 8 The $I-V$ characteristics of $\mathrm{AlGaAs} / \mathrm{InGaAs} / \mathrm{GaAs}$ PHEMTs with different temperature ranging from $300 \mathrm{~K}$ to $420 \mathrm{~K}$ in $30 \mathrm{~K}$ steps. 


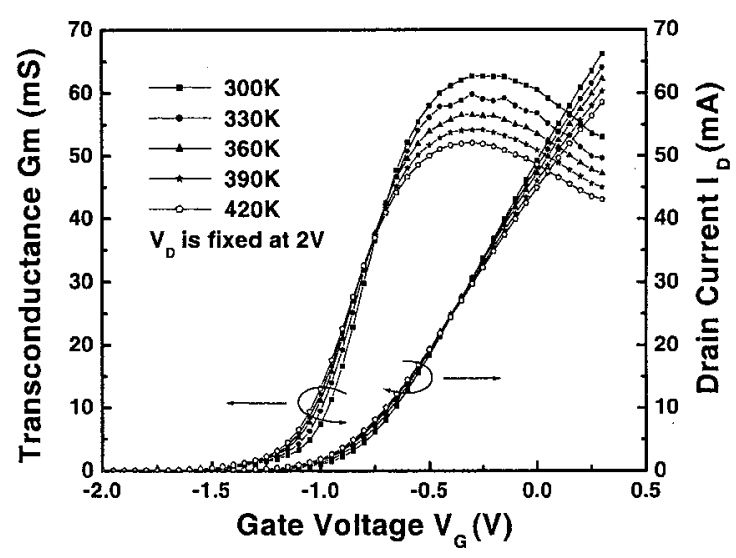

FIGURE 9 The transfer curve of AlGaAs/InGaAs/GaAs PHEMTs with different temperature ranging from $300 \mathrm{~K}$ to $420 \mathrm{~K}$ in $30 \mathrm{~K}$ steps.

temperature. It suggests that the scattering effects are more obvious with increasing temperature at higher $V_{G S}$ values than the effects of pinch-off voltage shift. This result may be that carriers in the channel increase with increasing $V_{G S}$ values, and the increase of the charge density induces the scattering effects increasing and mobility decreasing at higher temperature, which results in $I_{D}$ and $G_{m}$ degradation with increasing temperature at higher $V_{G S}$ values.

\subsubsection{Disappearance of the Kink}

Figure 8 shows that a kink is observed at $V_{D S} \sim 2.5 \mathrm{~V}$ at room temperature, indicating that trapping-detrapping phenomena are present [24-26]. However, the kink disappears at higher temperatures. A possible explanation is as follows: (1) At room temperature, when the drain voltage is applied, the electrons are accelerated by electrical field in the channel, and hotelectrons start injecting into adjacent layers and get trapped in deep levels. Therefore, the current reduction occurs because the number of available carriers in the active channel is decreased. As the drain voltage is further raised, the trapped electrons near the drain side are emitted, and the current increases again [27]. Thus, the kink appears at room temperature.

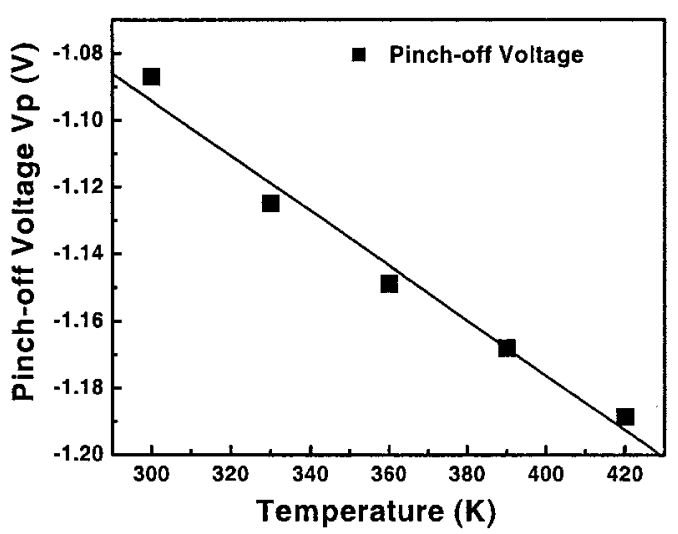

FIGURE 10 The pinch-off voltage versus temperature of AlGaAs/InGaAs/GaAs PHEMTs. 


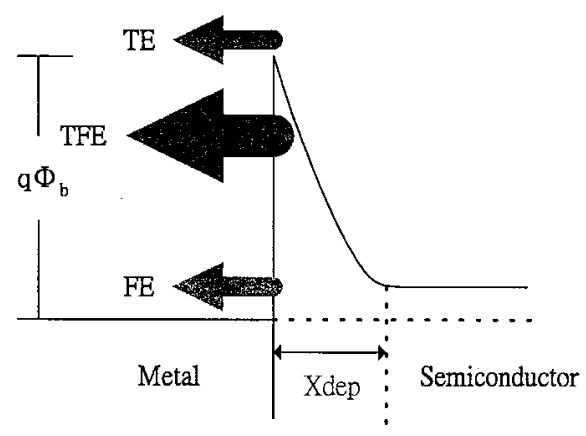

FIGURE 11 The mechanism of current transport in metal-semiconductor.

(2) At higher temperatures, the trapped electrons are energetic, and the traps are difficult to capture carriers. The capture/emission phenomena of traps then are less obvious at higher temperatures than at room temperature. Therefore, the kink disappears at higher temperatures.

\subsubsection{Thermal Effects of the Schottky Junction}

Experimental studies of metal-semiconductor contacts have shown that most of the metalsemiconductor combination form depletion layer contacts. The conduction properties of such contacts are determined by the actual transport mechanism, as shown in Figure 11, which can be due to:

(1) Thermionic Emission (TE): the carriers surpass the top of a barrier and give rise to rectifying behavior.

(2) Thermionic Field Emission (TFE): the tunnelling of hot carriers through the top of the barrier.

(3) Field Emission (FE): the carriers tunnel through the whole barrier.

(4) Recombination in the space.

TE and TFE are temperature-dependent (TFE and TE increase with increasing temperature), while FE is temperature-independent [21].

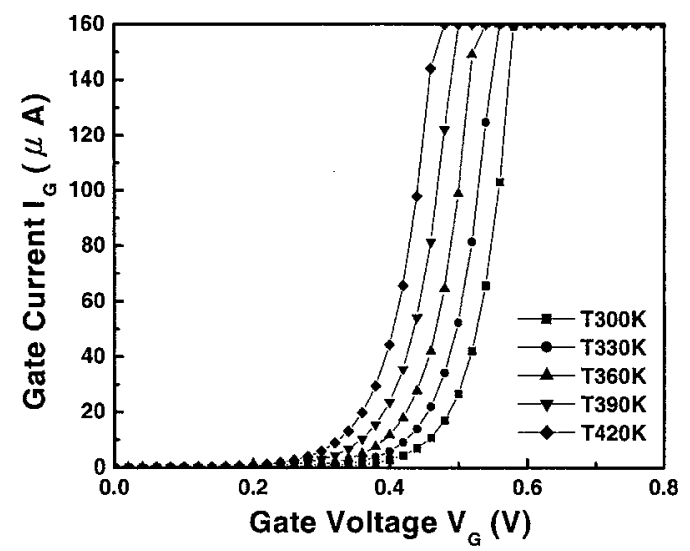

FIGURE 12 The forward-bias characteristics of gate-to-drain Schottky diode of AlGaAs/InGaAs/GaAs PHEMTs. 


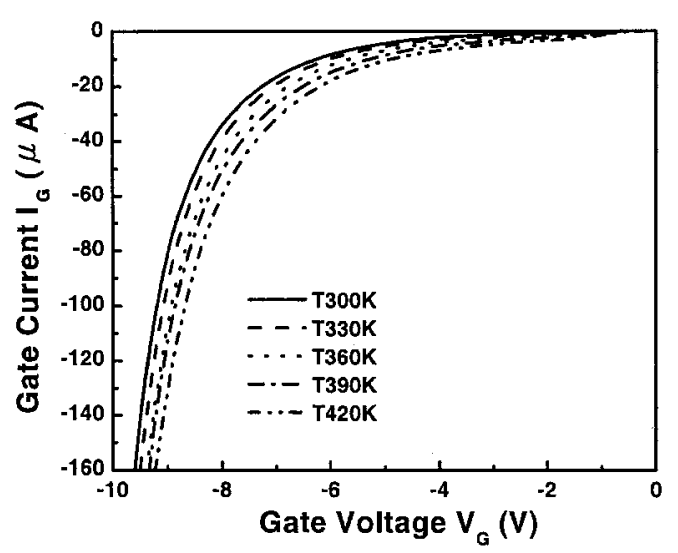

FIGURE 13 The reverse-bias characteristics of gate-to-drain Schottky diode of AlGaAs/InGaAs/GaAs PHEMTs.

The forward bias characteristics of the Schottky junction are shown in Figure 12, the turn on voltage reduces with increasing temperature, due to an increase of TE and TFE. Figure 13 shows the reverse bias characteristics of the Schottky junction, the gate leakage current tends to a point at higher $\left|V_{G S}\right|$ values. It suggests that the $\mathrm{FE}$ mechanism is more and more obvious with increasing $\left|V_{G S}\right|$ values. This result may be because the width of the depletion reduces at higher $\left|V_{G S}\right|$ values, causing larger tunnelling current, which results in the FE becoming a main mechanism at higher $\left|V_{G S}\right|$ values. Thus, the gate leakage current tends to a point at higher $\left|V_{G S}\right|$ values.

\section{SUMMARY}

In this report, the most important degradation mechanisms of AlGaAs PHEMTs (such as gate-drain breakdown walkout or the bell-shaped of $I_{G}$ ) are studied and discussed. In all of the devices under test the degradation mechanisms can be attributed to trap-related phenomena that can be split into two major categories: (1) the carriers trapped under the gate electrode or between $\mathrm{AlGaAs} / \mathrm{InGaAs}$ heterojunction after accelerated tests, that give rise to a shift of the threshold voltage $\left(\Delta V_{T}\right)$ or a degradation of $G_{m}$ and $I_{D S}$; (2) the creation of traps in the gate-drain region, resulting in a permanent increase of the gate-drain breakdown voltage.

In conclusion, experimental results indicate that hot-electron and temperature effects in AlGaAs PHEMTs should be carefully evaluated if the improvement of long-term reliability is needed.

\section{References}

[1] Dub, K. H. G., Liu, S. M. J., Wang, S. C., Ho, P. and Chao, P. C. (1993). IEEE 1993 Microwave and MillimeterWave Monolithic Circuits Symp. Dig., 99-102.

[2] Kasody, R. E., Dow, G. S., Sharma, A. K., Aust, M. V., Yamauchi, D., Lai, R., Biedenbender, M., Tan, K. L. and Alien, B. R. (1994). IEEE Microwave and Guided Wave Lett., 4(9), 303-304.

[3] Siddiqui, M. K., Sharma, A. K., Callejo, L. G. and Lai, R. (1999). IEEE MTT-S Digest, 951-954.

[4] Huang, P. P., Huang, T. W., Wang, H., Lin, E., Shu, Y. H., Dow, G. S., Lai, R., Biedenbender, M. and Elliot, J. (1997). IEEE Trans. on Microwave Theory and Tech., 45(12), Part 2, 2418-2423.

[5] Menozzi, R., Cova, P., Canali, C. and Fantini, F. (1996). IEEE Trans. Electron Devices, 43(4), 543-546.

[6] Ladbrooke, P. H. and Blight, S. R. (1988). IEEE Trans. Electron Devices, 35(33), 257-267. 
[7] Paccagnella, A., Zanoni, E., Tedesco, C., Lanzieri, C. and Cetronio, A. (1993). IEEE Trans. Electron Devices, 38(12), 2682-2684.

[8] Ladbrooke, P. H. and Blight, S. R. (1988). IEEE Trans. Electron Devices, 35(33), 257-267.

[9] Paccagnella, A., Zanoni, E., Tedesco, C., Lanzieri, C. and Cetronio, A. (1993). IEEE Trans. Electron Devices, 38(12), 2682-2684.

[10] Lee, K. W., Lee, K., Shur, M. S., Vu, T. T., Roberts, P. C. T. and Helix, M. J. (1985). IEEE Trans. Electron Devices, ED-32, 987-992.

[11] Rocchi, M. (1985). Physica 129B. North-Holland, Amsterdam, pp. 119-138.

[12] Thomasian, A., Saunders, N. L., Hipwood, L. G. and Rezazadeh, A. A. (1989). Electronics Lett., 25(11), $738-739$.

[13] Thomasian, A., Rezazadeh, A. A., Everard, J. K. A. and Hipwood, L. G. (1990). Electronics Lett., 26(14), 1094-1095.

[14] Canali, C., Cova, P., Bortoli, E. D., Fantini, F., Meneghesso, G., Menozzi, R. and Zanoli, E. (1995). The Technical Digest of IEEE IRPS, 205-211.

[15] Meneghesso, G., Canali, C., Cova, P., De Bortoli, E. and Zanoni, E. (1996). IEEE Electron Dev. Lett., 17, $232-234$

[16] Tedesco, C., Manfredi, M., Paccagnella, A., Zanoni, E. and Canali, C. (1991). IEDM Tech. Dig, 437-440.

[17] Zanoni, E., Manfredi, M., Bigliardi, S., Paccagnella, A., Pisoni, P., Tedesco, C. and Canali, C. (1992). IEEE Trans. Electron Devices, 39(8), 1849-1857.

[18] Schoen, K. J., Woodall, J. M., Cooper, J. A. and Melloch, M. R. (1998). IEEE Trans. Electron Devices, 45, $1595-1604$.

[19] Sze, M. (1981). Physics of Semiconductor Devices. Wiley, New York.

[20] Kuang, J. B., Tasker, P. J., Wang, G. W., Chen, Y. K., Eastman, L. F., Aina, O. A., Hier, H. and Fathimulla, A. (1988). IEEE Electron Device Lett., 9, 630-632.

[21] Piotrowska, A., Guivarc'h, A. and Pelous, G. (1983). Solid State Electronics, 26(3), 179-197.

[22] Rao, M. A., Caine, E. J., Kroemer, H., Long, S. I. and Babie, D. I. (1986). J. Appl. Phys., 61(2), 643-649.

[23] Chan, Y. J. and Pavlidis, D. (1994). IEEE Trans. Electron Devices, 41, 637-642.

[24] Okamoto, Y., Matsunaga, K., Kuzuhara, M. and Kanamori, M. (1997). IEEE MTT-S Dig., 3, 1191-1194.

[25] Fujita, S., Noda, T., Wagai, A., Nozaki, C. and Ashizawa, Y. (1993). Proc. 5th Int. Conf. Indium Phosphide and Related Materials, Paris, France, April 1993, pp. 497-500.

[26] Chao, P. C., Tessmer, A. J., Duh, K.-H. G., Ho, P., Kao, M.-Y., Smith, P. M., Ballingall, J. M., Liu, S.-M. J. and Jabra, A. A. (1990). IEEE Electron Device Lett., 11(1), 59-62. 

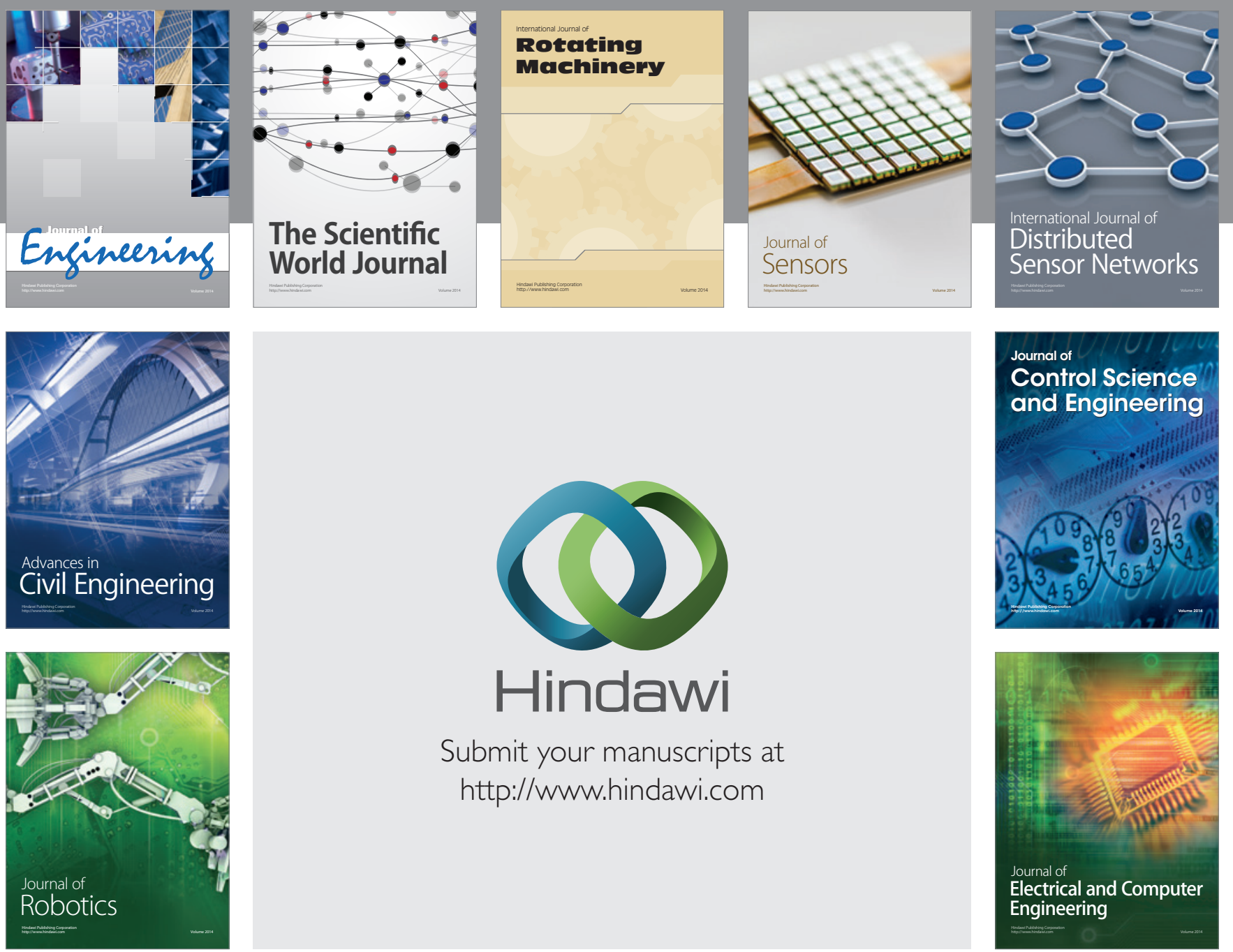

Submit your manuscripts at

http://www.hindawi.com
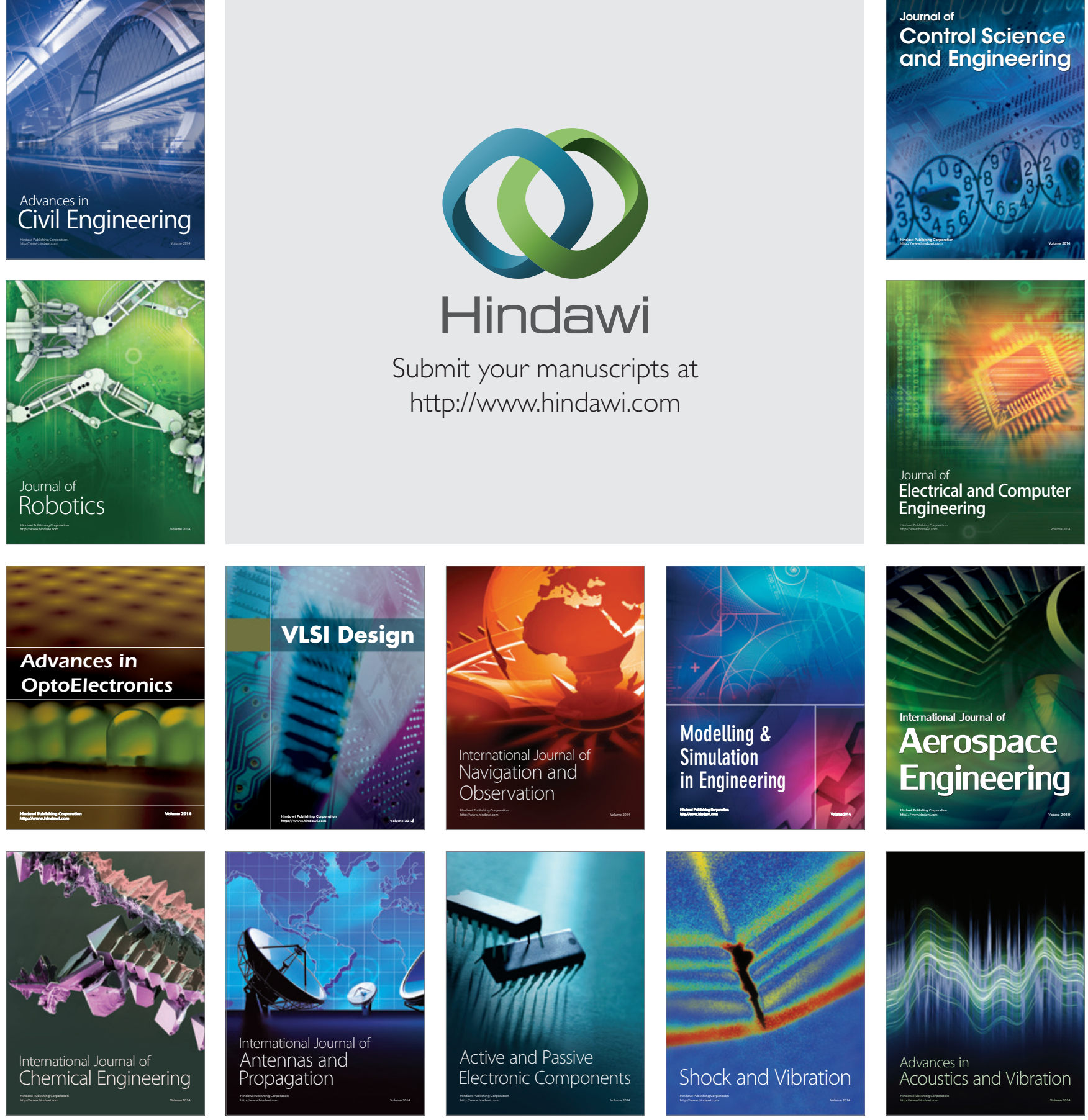